\title{
DOBRAS CUTÂNEAS EM ESCOLARES DE SETE A ONZE ANOS*
}

\author{
Raymond Victor Heger **
}

\begin{tabular}{l} 
RSPUB9/405 \\
\hline
\end{tabular}

Herio, R. V. Dobras cutâneas em escolares de sete a onze anos. Rev. Saúde púb!., S. Paulo, 12:147-50, 1978.

RESUMO: Análise do comportamento, em função de sexo e idade, das medida. de duas dobras cutâneas em 717 escolares de 7 a 11 anos, caucasóides, brasileiros, pertencentes a grupo sócio-econômico médio alto. Os valores médios das duas dobras cutâneas apresentaram acentuada e progressiva elevação em funçào da idade no sexo feminino e pouca oscilaça ro sexo masculino; foram mais elevados no sexo feminino com significancia ao nivel de $1 \%$ e a dobra tricipital sobrepujou a subescapular nos dois sexos e em todas as idades.

Uniterhos: Biometria. Composição corporal Crescimento fisico.

I TT T O DUCA O

A percentagem de gordura corporal pode ser avaliada por diversos métodos; assim, entre outros, usa-se medir a camada gordurosa nos diversos segmentos dos membros e no tronco em chapas radiográficas dos mesmos, ou então obter-se uma estimativa da gordura corporal através da análise de trocas bioquímicas envolvidas no metabolismo da gordura, ou ainda pela determinação da censidade do corpo. De maneira geral, tais métodos são complexos e dispendiosos e requerem técnicos, aparelhagem e instalações adequadas o que, dentro de certos limites, restringe sua utilização rotineira, principalmente quando se procura utilizá-los em coletividades; por outro lado, muitos são de execução extremamente difícil, senão inexequível, em se tratando de crianças.
A gordura corporal pode, contudo, ser avaliada de maneira satisfatória através da medida da espessura de dobras cutàneas que pode ser determinada em diversas localizaçóes no corpo e com auxilio de calibres apropriados. Mayer + afirma que tais medidas, quando comparadas com resultados fornecidos por outros métodos, dāo boa indicação não somente da gordura subcutânea, que representa cerca de $50 \%$ da gordura total, mas também desta última.

Este trabalho tem por finalidade mostrar o comportamento da espessura de duas dobras cutâneas em crianças e as variações condicionadas por sexo e idade.

* Resumo apresentado à 29a Renião da Sociedade Brasileira para o Progresso da Ciência em São Paulo. 1977 e no IV Congresso Brasileiro de Medicina Desportiva - Recife, 1977.

* Do Departamento de Ciências Sociais da Faculdade de Filosofia, Letras e Ciencias Humanas da USP - Cidade Universitária - 05508 - Säo Paulo - SP - Brașil e da Escola de Educação Física da USP - São Paulo - S? - Erasil. 
HEGG, R. V. Dobras cutâneas em escolares de sete a onze anos. Rev. Saúde públ., S. Paulo, $12: 147-50,1978$

\section{MATERIAL E MÉTODOS}

Os presentes dados foram obtidos durante o 2. semestre de 1972 e $1^{\text {o }}$ semestre de $1973 \mathrm{em}$ todos os escolares que cursavam as quatro primeiras séries dos Colégios Nossa Senhora de Sion e Pio XII na cidade de São Paulo. Os 717 escolares que compunham essa amostra eram todos caucasóides, brasileiros, com idade variando de 7 a 11 anos, sadios de acordo com us critérios clínicos gerais e pertencentes ao grupo econômico médio alto.

Os grupos etários foram constituídos levando-se em consideração a data do nascimento mais ou menos seis meses e em função da data na qual foi tomada a medida.

A dobra cutânea tricipital foi medida na face dorsal do braço, sobre o triceps, a meia distância entre o bordo externo do acrômio e o olecrano, no sentido do maior eixo do braço; a dobra cutânea subescapular logo abaixo do vértice inferior da escapula numa linha ligeiramente inclinada segundo a linha de clivagem natural da pele, conforme preconisam Tanner e Whitehouse. ${ }^{i}$ As duas dobras cutâneas foram determinadas com 0 Harpenden Skinfold caliper $(10 \mathrm{gr} / \mathrm{mm} 2)$ no lado esquerdo do corpo estando as crianças na posição de pé, ombros na posição de relaxamento natural e os membros superiores pendentes ao longo do corpo.

As diversas medidas são analisadas em termos de amplitude da variação, média aritmética, desvio padrão e coeficiente de variação.

\section{RESULTADOS}

As Tabelas 1 a 4 mostram os valores encontrados para as duas dobras cutâneas em função da idade e do sexo.

T A B E L A 1

Dobra cutânea tricipital em mm - sexo masculino.

\begin{tabular}{c|c|c|c|cc}
\hline idade & No & ampl. variação & média & d.p. & coef. variação \\
& & & & \\
7 & 46 & $6,60-21,40$ & 11,36 & 4,21 & 37,06 \\
8 & 51 & $5,40-21,60$ & 12,22 & 3,58 & 29,30 \\
9 & 43 & $5,60-18,60$ & 11,45 & 3,24 & 3,31 \\
10 & 44 & $6,60-23,60$ & 12,15 & 4,30 & 33,76 \\
11 & 25 & $6,20-23,40$ & 12,56 & 4,24 & \\
\hline
\end{tabular}

T A B E L A 2

Dobra cutânea tricipital em $\mathrm{mm}$ - sexo feminino.

\begin{tabular}{|c|c|c|c|c|c|c|}
\hline idade & No & ampl. & variação & média & d.p. & coef. variação \\
\hline 7 & 89 & 8,8 & $-28,8$ & 13,93 & 3,93 & 28,21 \\
\hline 8 & 106 & 8,4 & - $\quad 25,26$ & 14,61 & 4,10 & 28,06 \\
\hline 9 & 129 & 6,4 & -30.8 & 16,20 & 4,52 & 27,90 \\
\hline 10 & 127 & 8,4 & $-27,2$ & 16,29 & 4,09 & 25,11 \\
\hline 11 & 57 & 8,8 & $-29,0$ & 17,01 & 4,96 & 29,16 \\
\hline Total & 508 & & & & & \\
\hline
\end{tabular}


HEGG, R. V. Dobras cutâneas em escolares de sete a onze anos, Rev. Saúde públ., S. Paulo, $12: 147-50,1978$.

TABELA

Dobra cutânea subescapular em $\mathrm{mm}$ - sexo masculino.

\begin{tabular}{cc|c|cc|c}
\hline idade & $N^{\circ}$ & ampl. variação & média & d.p. & coef. variação \\
\hline & & & & & \\
8 & 46 & $4,00-17,20$ & 6,89 & 3.02 & 43,83 \\
9 & 51 & $3.60-19,40$ & 6,50 & 3,00 & 46,15 \\
10 & 43 & $4,20-14,60$ & 6,27 & 2.37 & 37,80 \\
11 & 44 & $4,20-16,60$ & 6,27 & 2,96 & 47,21 \\
\hline Total & 25 & $4,20-17.40$ & 7,12 & 3,54 & 49.72 \\
\hline
\end{tabular}

T A B E L A 4

Dobra cutânea subescapular em $\mathrm{mm}$ - sexo feminino.

\begin{tabular}{|c|c|c|c|c|c|c|}
\hline idade & $\mathbf{N}^{0}$ & ampl. & variação & média & d.p. & coef. variação \\
\hline 7 & 89 & 4,00 & $-21,00$ & 7,64 & 3,34 & 43,72 \\
\hline 8 & 106 & 4,20 & $-\quad \Sigma 2,80$ & 8,42 & 4,11 & 48,81 \\
\hline 9 & 129 & 4,20 & $-27,20$ & 9,79 & 5,08 & 51,89 \\
\hline 10 & 127 & 4,20 & $-\quad 21,40$ & 9,63 & 4,77 & 49,53 \\
\hline 11 & 57 & 4,80 & $-27,60$ & 10,61 & 5,97 & 56,27 \\
\hline Total & 568 & & & & & \\
\hline
\end{tabular}

\section{DISCUSSÃO}

O exame das Tabelas 1 a 4 mostra uma nitida, acentuada e progressiva elevação, em função da idade, dos valores médios das duas dobras cutâneas no sexo feminino o mesmo não se verificando no sexo masculino, no qual ambos os valores médios oscilam pouco e nos dois sentidos, com uma diferença para mais, considerando as idades extremas, de $1,20 \mathrm{~mm}$ para a tricipital e $0,23 \mathrm{~mm}$ para a subescapular. Por outro lado, os valores médios do sexo feminino são maiores que os do sexo masculino e, em ambos os sexos, a dobra tricipital sobrepuja a subescapular. O teste $t$ mostrou haver significância ao nivel de $1 \%$, em função do sexo, para as duas dobras e em todas as idades com exceção da dobra subescacular aos 7 anos.
Nossos dados aproximam-se dos apresen. tados por Malina 3 referentes a crianças brancas de Philadephia e pertencentes a classe sócio-econômica média para alta, e diferem bastante dos padröes dados por Hammond ", Ferro Luzzi e col. ${ }^{1}$ e Pett e col. ${ }^{5}$ possivelmente pelo fato de termos medido crianças pertencentes a uma amostra selecionada e portanto não representativa da coletividade em geral. Os dados de Sabharwal e col. ${ }^{6}$ referentes a meninas de grupo sócio-econômico alto situam-se numa posição intermediária entre os nossos e os dos outros autures citados.

\section{CONCLUSÓES}

Os altos valores médios das duas dobras cutâneas devem ser atribuidos à elevada 
HEGG, R. V. Dobras cucaneas em esculares cie sete a onze anos. kev. sumb públ, S. Paulo, $12: 147-50.1978$.

condição sócio-econômica do grupo analisado.

A diferenciação sexual é evidente em ambas as medidas das dobras cutâneas nas idades consideradas, menos aus 7 anos para a subescapular.

A dobra tricipital apresenta valores mais elevados que a subescapular nos dois sexos.

HEGG, R. V. [Skirfolds in 7 to 11 years old school children] Rev. Saúde públ., S. Paulo, $12: 147-50,1978$.

ABSTRACT: The correlation between two skinfolds of 717 calcasian Brazilian school children, 7 to 11 years old belorgirg to medium high socio-economic group was analyzed. The medium values of both skinfolds showed marked and progressive increase, by age, for females and little change for males. Skinfold measures for females were higher than for males, significant at the $1 \%$ level. The triceps shinfold was higher than the subscapular skinfold for both sexes for all ages.

UNITERMS: Biometry. Body composition. Growth.

\section{REFERENCIAS BIBLIOGRAFICAS}

1. FERRO-LUZZI, A. \& FERRO-LUZZI, G. Study on skinfold thickness of schoolchildren in some developing countries. I -- Skinfold thickness of Lybian boys. Metabolism, 11:1064. 1962.

2. HAMMOND, W. $H$. Measurement and interpretation of subcutaneous fat with norms for children and young adult males. Brit. J, prev. Med., 9:201-11, 1955.

3. MALINA, R. M. Patterns of development in skinfolds of negro and white Philadelphia children, Hum. Biol., 38:89-108, 1966.

4. MAYER, J. Some aspects of the problems of regulation of food intake and obesity. New Engl. J. Med., 2r4:6106. 1966.
5. PETT, L, B. \& OGILVIE, G. F. The canadian weight-height survey, Hum. Biol, $28: 177-88,1956$.

6. SABHARWAL, K. P. et al. Body measurements and creatinine excretions among upper and lover socio-economic groups of girls in Guatemala. Hum. Biol., 38:131-40, 1966.

7. TANNER, J. M. \& WHITEHOUSE, R. H. Standards for subcutaneous fat in British Children: percentiles for thickness of skinfolds over triceps and below scapula. Brit. med, J, 17:44650, 1962 .

Recebido para publicação em 21/07/1977 A provado para publicação em 25/10/1977 